\title{
Algunos desafíos curriculares de la formación ciudadana y la diversidad en Chile
}

\author{
MANUEL SILVA ÁGUILA* \\ MÓNICA LLAÑA MENA** \\ FABIOLA MALDONADO GARCÍA*** \\ ANDREA BAEZA REYES ${ }^{* * * *}$ \\ Universidad de Chile
}

Recibido el 20-09-2017; primera evaluación el 08-03-2018; segunda evaluación el 03-04-2018; tercera evaluación el 05-06-2018; aceptado el 30-06-2018

\section{RESUMEN}

El trabajo aborda la compleja relación entre la selección cultural del currículum que sostiene los planes y programas de estudio y la naturaleza diversa de las sociedades donde se inserta. Siguiendo una aproximación cualitativa, analizamos los discursos de docentes de la Región Metropolitana, acerca de sus representaciones de lo político y la política y sobre la importancia del currículo y sus posibilidades para la atención a la diversidad desde la formación en ciudadanía. Los resultados indican falta de problematización de la diversidad en esta materia. Nuevos fenómenos

\footnotetext{
* Profesor de Estado en Historia y Geografía, Universidad de Chile. Máster en Educación. Universidad de Lovaina (KUL) Bélgica. Doctor en Educación. Universidad Complutense de Madrid. Especialista en el área de Teoría del Currículum y Diseńo Curricular. Profesor de Pre y Postgrado en el Departamento de Educación, Facultad de Ciencias Sociales (FACSO), Universidad de Chile. Docencia y dirección de tesis en el área. Investigación y publicaciones en el área de educación y currículum. Correo: msilvaag@ uchile.cl

** Socióloga y magíster en Educación, Universidad de Chile. Académica del Departamento de Educación, de la Facultad de Ciencias Sociales (FACSO), Universidad de Chile. Investigadora en el área de Cultura escolar, resistencia y conflictos en la convivencia escolar e interculturalidad. Especialista en investigación educacional. Encargada de la cátedra de Teoría Socio antropológica aplicada a la Educación y Metodologías cualitativas. Correo: mllana2431@gmail.com

*** Pedagoga en Historia, Geografía y Educación Cívica, Universidad Austral de Chile. Socióloga, Universidad de Chile y candidata a doctora en Educación, Universidad Academia de Humanismo Cristiano. Académica de la Universidad de Chile, Facultad de Ciencias Sociales, Departamento de Educación. Temas de investigación: ciudadanía juvenil, convivencia escolar, desarrollo profesional docente. Correo: fmaldona@uchile.cl

**** Socióloga, Universidad de Chile. Asistente de investigación, Departamento de Sociología, de la Facultad de Ciencias Sociales (FACSO), Universidad de Chile. Asistente de investigación y publicaciones, Centro de Desarrollo Cognitivo, Universidad Diego Portales. Temáticas de investigación: género, comunicaciones y educación. Correo: andreaa.baeza@gmail.com
} 
contemporáneos, como la valoración de la formación ciudadana y la diversidad, exigen una mayor atención en los procesos de diseño curricular actuales para la convivencia democrática.

Palabras clave: currículo, educación para la diversidad, educación inclusiva, educación cívica

\section{Some curricular challenges of citizen training and diversity in Chile}

\section{Abstract}

The article analyzes the complex relationship between cultural selection of curriculum and the diverse nature of the societies in which it is inserted. From a qualitative approach, we analyze the teachers' discourses of the Metropolitan Region about their representations on the political issues and the Politics and on the importance of curriculum and its possibilities for the attention to the diversity from the formation in citizenship. Results show the lack of problematization of diversity in this area. New contemporary phenomena such as valuing diversity require greater attention in the current processes of curriculum design for democratic coexistence.

Keywords: curriculum, diversity education, inclusive education, civic education

\section{Alguns desafios curriculares da formaçáo cidadá e a diversidade no Chile}

\section{RESUMO}

O trabalho aborda a complexa relação entre a seleção cultural do currículo que sustenta os planos e programas de estudo e a natureza diversa das sociedades onde este se insere. A partir de uma abordagem qualitativa, analizamos os discursos de docentes da Regiáo Metropolitana sobre suas representaçóes do político e da política, e sobre a importância do currículo e suas posibilidades para a atenção à diversidade a partir da formação em cidadania. Os resultados indicam falta de problematização da diversidade nessa materia. Novos fenômenos contemporâneos, como a valoração da formaçáo cidadã e a diversidade, exigem uma maior atenção nos processos de desenho curricular atuais para a convivência democrática.

Palavras chave: currículo, educação em diversidade, educação inclusiva, Educação Cívica 


\section{INTRODUCCIÓN}

La escolaridad actual está tensionada por dos procesos que operan de manera simultánea. En Chile, existen algunos cambios en las políticas que buscan «normar el quehacer educativo» para atender la diversidad de los y las estudiantes (Muñoz, López y Assaél, 2015). En este marco, adquieren relevancia los crecientes procesos de inmigración, reflejo de la globalización, para el aseguramiento de la equidad educativa ante sujetos con diferencias socioeconómicas, de origen étnico/cultural, de género, entre otras. Luego, encontramos la aguda desafección frente a las obligaciones ciudadanas al interior de los sistemas políticos democráticos de los ciudadanos.

Frente a estos acuciantes desafíos, la gestión de las instituciones educativas debe proveer de herramientas a sus comunidades educativas, comprendiendo la naturaleza de cada uno de estos fenómenos en sus estructuras visibles e invisibles. También debiese proponer, de manera general, criterios que habiliten a quienes toman decisiones en la esfera educacional, adecuando las pautas de la organización en la aplicación de actuaciones que garanticen el respeto a la dignidad de todos los sujetos, además del desarrollo de una conciencia cívica que sustente una convivencia acorde con los valores esenciales de la humanidad en esta etapa del desarrollo histórico.

Hablar de la escuela es hablar de ciudadanía, pues sus profesores/as «tienen el compromiso de reconocer las diferencias combatiendo la desigualdad» (Kramer, 2005, p. 44). En ese sentido, las propuestas curriculares han de aceptar la diversidad cultural y reconocerla como riqueza para generar cambios hacia una sociedad más justa y democrática. Por lo mencionado, en este trabajo, comunicamos una aproximación inicial a este complejo tema. Para ello hemos hecho una indagación exploratoria, a través de los discursos de actores educativos de la educación media chilena, en una muestra acotada de los mismos.

\section{MARCo TEÓRICO}

\section{Diversidad y ciudadanía}

El crecimiento de los Estados nación, el debilitamiento de las fronteras, sumado al aumento de la inmigración, hacen surgir preguntas complejas sobre las nociones de diversidad y ciudadanía en un mundo global (Giddens, 1993).

Los Estados naciones se incrementaron de 43 en 1900 a 193 en 2015, como miembros de Naciones Unidas. El número de población viviendo fuera de sus 
países de origen equivalente a 120 millones en 1990, creció a 160 millones en 2000 y a 244 millones en 2015. De ellos, 26 millones son latinoamericanos (United Nations, 2016). Esto genera tensiones para los Estados, puesto que necesitan desplegar más ampliamente las políticas de apoyo en el desarrollo social: más hospitales, jardines infantiles, escuelas, universidades, etcétera.

Por su parte, Chile, actualmente, experimenta un alto número de migrantes viviendo dentro de sus fronteras. Se estima que hay cerca de 411.000 migrantes residentes permanentes, cifra que se ha duplicado entre 2002 y 2014. A partir de entonces, el flujo de inmigrantes ha sido reciente y expansivo, llegando a las escuelas e instalándose como tema y como desafío. En este contexto, es la concentración territorial de la población en algunas ciudades y comunas, y el arribo de nuevos flujos migratorios racializados aquello que incide en la representación colectiva que el país se está «llenando» de migrantes (Rojas y Silva, 2016). No obstante, la migración sigue siendo un bajo porcentaje $(2,3 \%)$ en comparación a los países desarrollados que superan el $11 \%$ con relación a la población nacional.

Desde esta perspectiva, es relevante también considerar los matices que develan otras esferas de las vivencias de quienes son sujetos de estos macroprocesos globales. En el caso de Chile, Tijoux y Córdova (2015) avanzan en dilucidar las subjetividades que se tensionan en los ámbitos de la racialización y sexualización, personificados en el cuerpo de las y los inmigrantes. Esto posibilita captar otra dimensión de la construcción de identidades nacionales y su inserción activa, como ciudadanos/as, en la vida de las sociedades que las acogen.

La migración —una expresión más de la diversidad — genera tensiones de todo orden en el concierto de los Estados en su capacidad de responder a través de las políticas en las distintas áreas. Como señala Monzón (2015), las políticas públicas debiesen tener un comportamiento estratégico o intencional a partir de la identificación de necesidades de cada grupo social, como expresión de la competencia de un gobierno. En ese sentido, es preciso poner a disposición de las diversidades de la población las mismas oportunidades y bienestar para generar desarrollo social en equidad.

Por ello, es importante remirar cómo el currículum puede ser capaz de atender a la diversidad, por la importancia que suscita para el desarrollo de ciudadanía y convivencia democrática. En esta dirección, es preciso poner en el centro de la preocupación curricular las formas de inserción o inclusión que se deben desplegar para compartir el espacio político común. De allí la necesidad de abrir la construcción de ciudadanía, que es, por definición, «una manera de tratar a la gente como individuos dotados de derechos iguales ante la ley» (Kymlicka y Norman, 2002, p. 18). 
Los criterios de selección cultural funcionales a esta apertura de la ciudadanía podrían implicar, por ejemplo, incluir las virtudes cívicas denominadas por Galston (1991) como ejes curriculares formativos. Estas son:

(a) virtudes generales: coraje, respeto de la ley, lealtad; (b) virtudes sociales: independencia, apertura mental; (c) virtudes económicas: ética del trabajo, capacidad de postergar las gratificaciones, adaptabilidad al cambio económico y tecnológico; y (d) virtudes políticas: capacidad de reconocer y respetar los derechos de los demás, disposición a no exigir más de lo que se puede pagar, capacidad de evaluar el desempeño de quienes ocupan los cargos públicos, disposición a participar en el debate público (pp. 221-224).

Mientras los elementos de este nuevo mundo diverso se vinculan al crecimiento de la migración internacional, también revelan el reconocimiento de desigualdades estructurales entre y dentro de los Estados.

Si observamos el índice de Gini que mide los coeficientes de desigualdad a nivel macrosocial, la mayoría de los países de la región avanza poco para reducir las diferencias de oportunidades en términos de ingreso y bienestar. Mientras que los países desarrollados tienen índices con valores cercanos a 0.23 - y tienden al 0 o la perfecta igualdad—, nuestros países sudamericanos obtienen un valor de 0.53 - más cercano a 1 - lo que implica una dirección hacia la desigualdad.

La diversidad impacta en todos los niveles del sistema educacional, puesto que, como fenómeno, establece una conexión entre la estructura social y las adscripciones a capitales culturales y sociales. Pero no siempre hay sintonía entre los códigos simbólicos de la escolaridad con el conjunto de la estructura social, sino que hay mayor cercanía con los estratos altos, como sostiene la teoría de los códigos sociolingüísticos de Bernstein (Díaz, 1985).

Siguiendo este enfoque, la diversidad se expresa en los códigos lingüísticos que median las interacciones al interior de los procesos de enseñanza, aprendizaje y desarrollo humano. Quienes poseen, conjuntamente, códigos restringidos y elaborados pueden tener una relación más fluida con las simbolizaciones, abstracciones, categorizaciones propias de la escuela y la universidad, no así quienes poseen solo el código restringido, más cercanos a lo concreto, contextual y de poco léxico.

El capital lingüístico no evidencia relación con la capacidad intelectual o la inteligencia; por ello, promover a cualquier estudiante a los códigos elaborados es un desafío ético para quienes ejercen la docencia en todos los niveles de la escolaridad, sobre todo en contextos crecientemente desiguales. El dominio lexicológico y semántico permite construir más sinergias neuronales y mayor capacidad de construir significados y sentidos con relación a los contenidos 
incluidos en el currículum prescrito. De esta forma, quienes realizan docencia o hacen gestión académica deberían generar, más ampliamente, programas de nivelación o propedéuticos, con el propósito de equipar con competencias de entrada adecuadas a quienes ingresan a la escolaridad, en cualquiera de sus niveles.

Pasar de la alfabetización a la construcción de significados comprensivos es un paso sustantivo a una inserción lúcida en los desafíos de la educación formal. Para lograr justicia educacional con la diversidad y que se haga cargo de este desafío ético, es necesario proveer, a los estudiantes, andamiajes cognitivos mediados por el lenguaje para construir aprendizajes significativos. Esto es fundamental para pensar la ciudadanía y actuar en ella.

\section{Currículum y ciudadanía}

Uno de los desafíos más acuciantes para el currículum en la educación formal, al interior de un Estado, es ayudar a tomar conciencia de las complejas relaciones que se establecen entre la unidad y la diversidad, tanto en sus comunidades locales, regionales, nacionales como con el mundo global (Beck, 1998; Silva, 2008).

Como entidad simbólica, el currículum escrito provee de coordenadas para conocer, orientar y valorar aspectos (contenidos de disciplinas del saber) seleccionados del mundo físico, natural, social y cultural, todas ellas enmarcadas en planes formativos con perfiles delimitados. Como plantea Pinar (2004), se trata de una conversación compleja donde, quienes tienen el poder de selección cultural, definen los contenidos de la misma en el proceso de transmisión cultural intencionado, otorgando contenido a la experiencia escolar. En la nomenclatura curricular de Glatthorn, Boschee, y Whitehead (2006), se distinguen los siguientes: currículum escrito (campo de los decisores curriculares), currículum apoyado (el sustrato material del escrito), currículum enseñado (la adecuación al contexto escolar de currículum escrito por parte de los docentes), y el currículum medido (encargado de constatar los aprendizajes, consecuencia de la enseñanza impartida). Por su naturaleza eminentemente política, y siguiendo a los autores, entre sus funciones centrales, están la mediación, la normalización y el control.

A saber, sus prescripciones median entre los ideales del currículum recomendado «que se desprende de las múltiples agencias de la sociedad civil o de los grupos de presión que buscan influir en la toma de decisiones sobre qué enseñar en el proceso de selección cultural, aspecto clave del currículumy las heterogéneas realidades de las aulas situadas en múltiples contextos 
políticos, económicos, étnicos, geográficos, lingüísticos, religiosos, de género, entre otros. Por tanto, representa un compromiso necesario y funcional entre lo que los expertos exhortan a enseñar y aquello que los docentes creen que se puede o debe enseñar. Lo mismo sucede entre las expectativas del sistema y de los equipos directivos de una institución y las preferencias múltiples de los profesores.

Desde el punto de vista de la normalización, se trata de construir un sustrato cultural básico mínimo común, que permita transmitir saberes y puntos de vista compartidos entre todos los sujetos en los ámbitos nucleares de la estructura social y del Estado del que forman parte. De esta manera se comparte una base cultural común.

La sujeción de las instituciones a la mediación y normalización permiten el control de los mensajes en la transmisión cultural intencionada en la escolaridad. Prueba de ello son las evaluaciones sistémicas institucionalizadas de carácter censal, tales como las pruebas SIMCE en Chile, Saber en Colombia, y Enlace en México. También se refleja en los sistemas de evaluación para el ingreso a la educación superior.

En Chile, ha habido esfuerzos permanentes desde el Estado por impulsar una formación cívica o ciudadana en la escuela, que permita la identificación de la nación con el régimen político republicano y el consecuente desarrollo de aquellas prácticas de sociabilidad y de convivencia en el espacio público que den cuenta de este vínculo (Mardones, 2015; Salazar y Pinto, 2010).

La formación para la ciudadanía que se ha desarrollado para la escuela en este país ha tenido diversos sellos y énfasis curriculares. En dictadura, el currículo de 1980 privilegió los conceptos de identidad, el patriotismo, nacionalismo y respeto a las instituciones, valorando la pertenencia a la comunidad política por sobre la participación. En democracia, en cambio, el currículo de 1998 valora la participación democrática y al ciudadano con derecho a participar en la generación del orden político y sus decisiones, con conocimientos y competencias que le permitan escrutar y enjuiciar tal orden y su legitimidad (Bascopé, Cox y Lira, 2015).

Preparar a los y las estudiantes en roles ciudadanos significa ayudarles en el desarrollo de conocimientos, comprensión y formación de actitudes positivas hacia la vida en ciudadanía. En el último tiempo, no solo ha habido un ensanchamiento de la noción de ciudadanía, sino también del rol clave de la «educación cívica y ciudadana» (ECC) para hacer frente a las demandas y desafíos de las sociedades en el siglo XXI (Schulz, Ainley, Frailon, Kerr y Losito, 2010). 
Si bien la ECC es reconocida como uno de los aspectos más importantes, existe una brecha entre esta declaración de principio y su implementación en las escuelas. Según el último International Civic and Citizenship Study (ICCS) de 2009, coexisten diferentes enfoques dentro de los sistemas escolares, aun cuando la legislación, regulaciones y un currículum común son establecidos a nivel nacional. En cuanto a la implementación, el ICCS 2009 revela que los docentes en Chile privilegian ampliamente la promoción de conocimiento acerca de los derechos y deberes ciudadanos por sobre la participación estudiantil o la preparación para la participación política futura (Schulz et al., 2010).

En un contexto de alto desinterés por el ejercicio de la política tradicional, particularmente el voto y la pertenencia a partidos políticos, junto con una creciente desconfianza hacia las instituciones de la democracia representativa y la misma clase política (Norris, 2003), cada vez son más los jóvenes que se involucran en formas emergentes de compromiso cívico, articuladas por fuera de la esfera institucional de la política. En contraste con la comprensión de la juventud apática, estudios recientes confirman que se mantiene el interés juvenil en la política y en los asuntos públicos (Amnå y Ekman, 2013; Sloam, 2016).

En el marco de estas nuevas formas de participación «no electoral» y «no institucionalizada», destacan las actividades juveniles basadas en temas/ problemas en estrecha relación con las propias experiencias y percepciones de la democracia y que les son cercanas a la vida cotidiana de las personas (Dalton, 2009), como son las luchas por demandas postmateriales. Estas formas de participación indican el desplazamiento de la política hacia lo político, cuya articulación ciudadana depende estrechamente de la cultura cívico-política específica de cada país (Sloam, 2016).

La educación no puede estar ajena a estos fenómenos de cambio y desconocer el involucramiento ciudadano en lo político (Dürr, 2004). En 2016, se establece en Chile la Ley 20.911 para que todos los establecimientos educacionales reconocidos por el Estado incluyan Planes de Formación Ciudadana para los niveles de educación parvularia, básica y secundaria. Estos deberán abarcar la gestión, la actividad curricular y extracurricular, con el propósito de que las y los estudiantes adquieran habilidades, conocimientos y actitudes que fortalezcan la ciudadanía (Mineduc, 2016). Más específicamente, se trata de un Plan de Formación Ciudadana

(...) que integre y complemente las definiciones curriculares nacionales en esta materia, que brinde a los estudiantes la preparación necesaria para asumir una vida responsable en una sociedad libre y de orientación hacia el mejoramiento integral de la persona humana, como fundamento del sistema democrático, la justicia social y el progreso (Mineduc, 2016, p. 16). 
Finalmente, educar es un proceso doble, que «estimula el desarrollo de las capacidades, aptitudes y vocaciones latentes y, al mismo tiempo, encausa su ejercicio para que sirvan a la conservación y fomento de aquellos valores éticos e intelectuales que (...) la sociedad estima como los mejores» (Labarca, 1944 , p. 9). Por ello, es menester reconocer la diversidad como desafío para el currículum que quiera profundizar la democracia y la justicia social (GonzálezValencia y Santisteban-Fernández, 2016).

Conforme a lo anterior, el presente escrito se enmarca en la Investigación interdisciplinaria «Representaciones de lo político en estudiantes y profesores en la educación secundaria de la Región Metropolitana (R.M) de Chile. Criterios para una nueva propuesta de formación ciudadana». Esta fue llevada a cabo por el Departamento de Educación de la Facultad de Ciencias Sociales y el Departamento de Estudios Pedagógicos de la Facultad de Filosofía y Humanidades de la Universidad de Chile entre 2014 y 2015. Además, contó con el apoyo y financiamiento de Iniciativa Bicentenario de la misma casa de estudio.

El objetivo general de la investigación fue comprender las representaciones respecto de lo político y la participación ciudadana en los estudiantes y en sus profesores de enseñanza media de la R.M. para generar bases de una propuesta en formación ciudadana. En particular, este escrito aborda el objetivo específico de indagar en la importancia del currículo y sus posibilidades para la atención a la diversidad desde la formación en ciudadanía en la escuela, tomando en cuenta los discursos docentes en este nivel educativo.

\section{Metodología}

El paradigma epistemológico adoptado fue la fenomenología, dada la complejidad de los fenómenos sociales y su impacto en la vida de los sujetos sociales, para nuestro caso de estudio, docentes de enseñanza secundaria. Las lecturas de la realidad social son acercamientos hacia un nivel de profundidad de su contenido simbólico, relevantes para la comprensión social de la formación ciudadana en la escuela vinculada al currículum.,

Así, bajo la idea de inmersión e incursión intensiva en la realidad del contexto escolar en el que sujetos intercambian significados en procesos interactivos, esta investigación tuvo un enfoque cualitativo, orientado a conocer, de manera profunda, la construcción de representaciones de lo político y los desafíos curriculares. El carácter de la investigación fue descriptivo-interpretativo, cuyo levantamiento de información se realizó en los años 2014 y 2015, previo a la promulgación de la Ley de Formación Ciudadana de 2016 (Biblioteca del Congreso Nacional [BCN], 2016). 
Como estrategias, se trabajó con entrevistas en profundidad y grupos de discusión. Previa autorización mediante consentimientos informados, se transcribió el material grabado en audio de estas conversaciones. La información fue organizada mediante unidades temáticas (Delgado y Gutiérrez, 1995; Mucchielli, 2011), las que fueron analizadas e interpretadas fenomenológicamente con el propósito de reconstruir la lógica que sostiene esas estructuras de sentido.

La decisión de emplear aquellas estrategias de investigación responde a que, en el habla, se articula el orden social y la subjetividad. De esta manera, es posible develar la ideología subyacente en los discursos concebidos como el conjunto de producciones significantes respecto a la democracia, la ciudadanía, lo político y la política, todas diseminadas en lo social y en la palabra, permitiendo desenmascarar dichas estructuras de sentido (Delgado y Gutiérrez, 1995).

\subsection{Muestra}

Participaron de esta investigación 4 docentes en entrevistas individuales (2 profesores y 2 profesoras). En los dos grupos de discusión participaron 6 y 7 docentes de ambos géneros respectivamente. Hubo diversidad en las disciplinas que impartían (humanistas, científicas), en la administración de las escuelas de la Región Metropolitana de Chile en las que trabajaban (municipales, particulares-subvencionadas y particulares) y en los años de experiencia profesional ejerciendo docencia escolar (entre 10 y 35 años). El criterio de selección de las y los docentes fue intencionado. Los sujetos que participaron en las entrevistas fueron distintos a quienes participaron en los grupos de discusión.

\subsection{Instrumento}

Fue elaborado un guion temático para realizar tanto las entrevistas como los grupos de discusión, el cual consideró 2 dimensiones claves: i) La Política y lo Político y ii) Escuela, y formación ciudadana. Los subtemas que se indican a continuación: 
Tabla 1. Guion temático para entrevistas y grupos de discusión

\begin{tabular}{|l|l|}
\hline La Política y lo Político & Concepto/Sentido \\
& Importancia \\
& Limitaciones \\
& Prácticas \\
& Conversación \\
\hline \multirow{2}{*}{$\begin{array}{l}\text { Escuela y formación ciu- } \\
\text { dadana }\end{array}$} & $\begin{array}{l}\text { Valoración } \\
\text { Profesores/profesor jefe } \\
\text { Experiencias democráticas } \\
\text { Poder/Decisiones } \\
\text { Espacio de reproducción /transformación } \\
\text { Imaginario de derechos y diversidad }\end{array}$ \\
\hline
\end{tabular}

\section{Resultados}

\subsection{Visión de la política, lo político: ¿Expresión de diversidad?}

Los y las docentes de la muestra distinguen entre la política y lo político. Perciben a la política «aún como espacio de transformación y de incidencia en la sociedad» y precisan que lo político, para el cuerpo docente, no se circunscribe solo a los partidos políticos, "es otra cosa, es movilizarse, es liderar y tomar decisiones; para mí la política es organizarse, pero organizarse con algo que a uno le genere sentido y a partir de esa organización construir un proyecto viable» (GD).

Aquí se hace visible la conformación del orden social, que enmarca y regula a los sujetos. En ese espacio emerge lo político y la política, en donde «lo político está vinculado al momento instituyente, y la política relacionada con la administración de lo instituido" (Retamozo, 2009, p. 70).

Existe reconocimiento que «desde (hace) un tiempo, el país está despertando y se reactivan las movilizaciones con ganas de hacer cosas en conjunto» (GD). En otras palabras, se observa que las manifestaciones sociales encarnarían 
las demandas para generar cambios a nivel de ciudadanía. Sin embargo, los profesores reiteran que «en lo cotidiano, no hay espacios para hacer política, para ejercer el derecho de toma de decisiones»(GD). Con ello, se seguiría reproduciendo la distancia entre las vidas privadas y el cotidiano escolar con lo que sucede en lo público politizado.

Existe un nudo crítico susceptible de inferir en esas expresiones discursivas: en oposición, en un nivel macro, está la sociedad que se moviliza, mientras que, en un nivel micro, la escuela es coercitiva. Por un lado, se expresa la conexión entre ciudadanía-democracia y entre ciudadanía-participación, lo que permite inferir que, desde esos imaginarios, «es importante el ejercicio ciudadano para aprender la democracia» (Entrevista). Por otra parte, si bien se expresa la crítica respecto al tipo de ciudadano que se estaría formando, donde «es necesario conocer esa formación» (Entrevista), no se explicita cual sería el aporte pedagógico al respecto.

\subsection{Currículum y formación ciudadana}

Respecto a la implementación curricular de la formación ciudadana, las y los profesores hacen notar sus críticas, estableciendo que «no hay [espacio para conformar] comunidad en la unidad educativa». En la escuela se realizan actividades aisladas porque "todo [está] fragmentado igual que el plan de estudios» (GD). Para ellos es necesaria esta formación e indican que «hace falta un ramo de «educación política» o "cívica» para desarrollar el pensamiento crítico» (GD) y la participación política. Insisten, además, en que no es bueno asumir modelos de otros países, enfatizando que el nuestro es "un modelo impuesto", donde los establecimientos «se centran en el desarrollo por competencias y no [se] estarían educando» en un sentido de formación integral (GD).

A pesar de que el camino de la formación ciudadana en la educación formal se inicia en la escuela, en el caso chileno, la discusión ha sido escasa en este escenario previo a la Ley en esta materia, la que busca desarrollar de una sociedad más justa y equitativa. Desde el punto de vista de las y los profesores, y sin tematizar sobre la diversidad en la escuela, hay un marco prescriptivo que limita el quehacer docente. Existe «falta de liderazgos, en las unidades educativas, los profesores no pueden salirse de lo prescrito" y se remarca cuando insisten en que el «sistema de trabajo está preestablecido y funciona así desde mucho tiempo» (GD). Por último, reconocen que existe una evaluación permanente sobre el sistema educativo y sus profesores, donde «la sociedad, los políticos y el ejecutivo dicen que lo que se hace [en la escuela] no está bien» (GD). 
Cabe señalar que, desde el punto de vista conceptual, la propuesta ministerial de Formación Ciudadana -invisible en los discursos producidos en este estudio- no se ajustaría a la comprensión de fenómenos complejos, como son los contextos de interacción en el marco de los espacios educativos, esto es, desde las instituciones más cercanas a su vida cotidiana, hasta aquellas en donde tienen lugar otros procesos socializadores. Los profesores reconocen también algunas debilidades, como la falta de diferentes opciones para definir el concepto de ciudadanía y de orientaciones para abordar la formación política de sus estudiantes, ya sea desde una aproximación clásica de ciudadanía o como una dimensión dentro de una concepción más amplia.

Finalmente, estos actores sociales proponen algunas estrategias para el desarrollo de la formación ciudadana. Para ello, subrayan la importancia que adquiere el rol de profesor jefe, quien genera un «vínculo más directo con los alumnos, más allá de lo escolar [y con este] se abordan otros temas, con la familia [...] Establece un vínculo emotivo en contraposición [con lo que en la escuela se exige el SIMCE, la PSU] y donde muchas veces lo valórico no tiene cabida [...], el rol de profesor jefe es muy gratificante»(GD). De este modo, se genera una mirada diferente del proceso educativo basado en una construcción mutua, de ahí esta representación positiva.

En la sala de clases los profesores que podrían ser futuros formadores son «aquellos que generan espacios democráticos, los que propician la participación, dan la palabra. Reconocen a los estudiantes como legítimos. Tratan de conocer sus necesidades, independientes de si son profesores jefes o no»(GD). En otros términos, habría un reconocimiento de la importancia del rol del profesor en la formación ciudadana y de las posibilidades reales de que se concretice en las aulas. Tal como los docentes señalaron, «el rol tradicional del profesor cambia, se transforma en promotor de conocimiento construido con los alumnos» (GD).

Puntualizando en una asignatura dentro del currículum, se reconoce la necesidad de formar en ciudadanía, pues «haría falta que existiera un ramo de educación política o educación cívica pero que de verdad orientara a los alumnos a tener un pensamiento crítico». Aunque se señala también que «en los colegios no hay una educación política, solo un modelo impuesto» (GD). Existirían algunas asignaturas, en opinión de las y los profesores del estudio, que facilitarían la realización de este tipo de actividades formativas como Educación Musical y Educación Física, porque provocarían una conexión distinta con los alumnos, puesto que «los ambientes de aprendizaje son diferentes, lo mismo que las temáticas» (GD). 


\section{DisCuSIÓN Y CONCLUSIONES}

A partir de los discursos analizados de la muestra de docentes de escuelas secundarias de la Región Metropolitana de Chile, se devela un nudo tensional en estos actores sociales partícipes de la vida social, en el cual, al no poder hablar de política, han elaborado construcciones simbólicas de lo político y la política, básicamente, desde el sentido común. En ese sentido, aún falta profundizar en cuáles son las limitaciones propias del ejercicio docente que vislumbran en la asunción de roles formadores en una sociedad sujeta a profundos y acelerados ritmos de cambio.

Un elemento por considerar para esta dimensión es la emergencia de la individuación, proceso por el cual las personas tienen que tomar decisiones respecto a su propia vida por sobre los factores estructurales y deben hacerse cargo de sí mismas (Araujo y Martuccelli, 2012; Martuccelli y de Singly, 2012; Touraine, 2001; Pérez, 2000). Siguiendo el discurso de los/as profesores/as del estudio, estos/as deben asumir individualmente sus responsabilidades y también los fracasos atribuidos a su gestión, por mencionar algunos, las exigencias de la escuela, el control de sus tiempos, la racionalidad instrumental que subyace a sus metas estandarizadas, la precariedad de un status que produce tensiones no asumidas institucionalmente. Todo ello genera una reflexividad que impide el desarrollo del pensamiento pedagógico y de decisiones profesionales autónomas acerca de los procesos formativos que legítimamente les competen.

El proceso de individuación enfrenta a los sujetos a la necesidad de desenvolverse en un espacio de requerimientos, de exigencias, en un ámbito de turbulencias multivariadas, y que los constriñe al extremo de relegarlos a un desempeño precario desde un punto de vista profesional. Cabe recordar que, en este proceso de subjetivación, el predominio de respuestas individuales a problemas colectivos también se relaciona con «la debilidad de las organizaciones sindicales que los agrupan y la existencia de contextos laborales flexibles y altamente precarizados» (Reyes-Jedlicki et al., 2014, p. 195).

Vinculado a lo anterior, se inscriben los hallazgos encontrados en una investigación actual sostenida en la narrativa de profesores chilenos de ciencias sociales de educación media (Cavieres-Fernández, 2017) quienes confrontan las políticas educacionales basadas en la rendición de cuentas y la estandarización con los constreńimientos de su rol en la enseñanza de la educación ciudadana. Tal como menciona Reyes-Jedlicki et al. (2014), finalmente, los docentes que se sitúan «en la disyuntiva de educar integralmente a sus estudiantes o prepararlos para que rindan exitosamente las pruebas estandarizadas» (p. 193). 
No deja de sorprender que la formación ciudadana, dentro del currículum, se siga considerando responsabilidad de una asignatura. Desde una perspectiva temporal, el currículum chileno desplazó su foco de la Educación Cívica desde una perspectiva asignaturista, hacia la educación ciudadana global. En palabras de Mardones (2015), esto implica añadir a los contenidos conceptuales de rango constitucional el «desarrollo de habilidades e internalización de valores concebida como una experiencia escolar integral» (p. 147).

En el contexto chileno de rezago democrático o de democracia incompleta (Garretón, 2010), los discursos de las y los docentes develan la persistencia de una comprensión tradicional en torno a la ciudadanía, que privilegia el componente intelectual por sobre el experiencial; es decir, que la reduce a la instrucción en vez de la aprehenderla como formación en su sentido más amplio (Álvarez, 2016). En esta línea, se requiere una perspectiva que abrace la «ciudadanía activa» y esté respaldada por «escuelas democráticas» que ofrezcan una "cultura participativa» (Eurydice, 2005 en Schulz, et al., 2010, p. 43) en y fuera de las escuelas.

Asimismo, llama la atención que no se tematice sobre la diversidad dentro de la escuela más allá de entenderla como la libertad de opinión y no como las complejidades culturales, sociales, religiosas, de identidad de género y orientación sexual. Podemos aventurarnos a señalar que la diversidad aún no constituía, en aquel entonces, un factor crítico, puesto que la misma formación ciudadana en el momento que se les preguntó a las y los docentes, no era una realidad formalizada, como lo es hoy con la Ley 20.911 sobre Planes de Formación Ciudadana (BCN, 2016). En este sentido, cabe interrogarse las razones que fundamentan el desconocimiento de los profesores de esas propuestas o bien cuáles fueron las razones que impidieron su concreción en la práctica curricular.

En los discursos docentes no está explicitada la diversidad del alumnado como un tema a considerar en su comprensión de lo político y formación ciudadana. Esto es un aspecto fundamental para que podamos vivir juntos en sociedad, puesto que la vida en comunidad política requiere del reconocimiento mutuo entre iguales y diferentes como sujetos (Touraine, 2001). Además, tiene relación con lo planteado por Castillo (2016): «la educación ciudadana en el aula depende mucho de las comprensiones y vivencias de los profesores, lo que trae mucha variabilidad entre las formas clásicas de enseñar la democracia» (p. 23). No es lo mismo comprender la diversidad desde un enfoque funcional o crítico, abordarla para algunos o para todos, acotarla a la educación o ampliarla como proyecto político (Ferrâo, 2010). 
La atención a la diversidad para una educación en equidad reconoce a la escuela como un espacio importante en la generación de oportunidades para «dejar marcas en los sujetos de una sociedad un poco más justa e igualitaria», así como también propiciar "una cultura que valora la diferencia y la promueve como base del enriquecimiento personal y social» (Dussel, 2004, p. 331). Siguiendo esta línea, «[...] la ciudadanía necesita aprender a aceptar, valorar y respetar su propia diversidad cultural, para luego ser capaz de apreciar la compleja y rica diversidad cultural que existe más allá de los bordes nacionales» (Barra, 2007, p. 18).

No es posible desconocer que, en los últimos veinte años Chile ha experimentado cambios profundos en su desarrollo político, económico, social y cultural, incluyendo un exponencial aumento de la inmigración, constituyendo una sociedad diversa que desafía el aparato cívico-político y educacional. Estas transformaciones han impactado de manera incuestionable al sistema educativo y, sin excepción, a todas las comunidades educativas que se están enfrentando a una nueva realidad diversa y multicultural. El contexto que enmarca un sistema social es complejo, cambiante y de incertidumbre (Bajoit, 2003; Bauman, 2004; Beck, 1992; Giddens, 1993), no solo para quienes enseñan, sino para quienes han de definir los contenidos y estrategias para abordar la diversidad (Pérez, 2000; Ferrâo, 2010).

Finalmente, el diseño curricular, entendido como el marco que regula la enseñanza, no debe contener solo la mirada de los expertos responsables de la elaboración del currículum escrito, como ocurre en la actualidad. Es preciso incluir la experiencia y saberes de los docentes de todos los niveles del sistema escolar, que, a través de la enseñanza, traducen y median sus prescripciones en el aula. De esta manera, se subraya el aporte de los docentes para la co-construcción de un currículum de formación ciudadana relevante y significativo, que incluya la diversidad, en consonancia con las nuevas realidades no solo políticas, sino también sociales y culturales.

\section{REFERENCIAS BIBLIOGRÁFICAS}

Álvarez, E. (2016). Es necesario visibilizar en los estudiantes no solamente la dimensión de alumnos, sino también la de ciudadanos. Entrevista. Docencia 58(1), 43-49.

Amnå, E. y Ekman, J. (2014). Stanby citizens: diverse faces of political passivity. 10.1017/S175577391300009X

Araujo, K. y Martucelli, D. (2012). Desafios comunes. Retrato de la sociedad chilena y sus individuos. Tomo I. Santiago de Chile. LOM. 
Bajoit, G. (2003). Le changement social.Approche sociologique des societés occidentales contemporaines. París. Armand-Colin Editeur.

Barra, N. (2007). El desafío de la diversidad cultural para Chile. Arlington, MA: Intercultural Center for Research in Education (INCRE). Recuperado de http://www.incre.org/pdf/Chile.pdf

Bascopé, M., Cox, C. y Lira, R. (2015). Tipos de ciudadanos en los currículos del autoritarismo y la democracia. En C. Cox y J.C. Castillo (eds.), Aprendizajes de la ciudadanía. Contextos, experiencias y resultados (pp. 245281). Santiago de Chile: Ediciones Universidad Católica de Chile.

Bauman, Z. (2004). Modernidad liquida. Buenos Aires: FCE.

Beck, U. (1992). La sociedad del riesgo: Hacia una nueva modernidad. Buenos Aires: Paidós.

Beck, U. (1998). ¿Qué es la globalización? Falacias del globalismo, respuestas a la globalización. Barcelona: Paidós.

Biblioteca del Congreso Nacional (BCN), Chile (2016). Crea el Plan de Formación Ciudadana para los establecimientos educacionales reconocidos por el Estado. Recuperado de https:/www.leychile.cl/ Navegar?idNorma=1088963

Castillo, J. (2016). Formar ciudanía en el Chile actual: una mirada a partir del desarrollo humano. Docencia, 58(1), 18-30.

Cavieres-Fernández, E. (2017). Teacher counter stories to a citizenship education mega policy narrative. Preparing for citizenship in Chile. Journal of Curriculum Studies, 49(4), 414-436. https://doi: 10.1080/00220272.2016.1274783

Dalton, R. (2009). The Good Citizen: How a younger generation is reshaping american politics. Washington DC: QC Press.

Delgado, J. y Gutiérrez, J. (1995). Métodos y Técnicas cualitativas de investigación en ciencias sociales. Madrid: Editorial Síntesis.

Díaz, M. (1985). Introducción al estudio de Bernstein. Revista Colombiana de Educación, (15), 5-17.

Dürr, K. H. (2004). The school: A democratic learning community. Estrasburgo, Francia: Council of Europe.

Dussel, I. (2004). Desigualdades sociales y desigualdades escolares en la Argentina de hoy: algunas reflexiones y propuestas. Buenos Aires: FLACSO.

Eurydice. (2005). Citizenship education at school in Europe. Bruselas, Bélgica: Autor.

Ferrâo, V. (2010). Educación intercultural en América Latina: distintas concepciones y tensiones actuales. Estudios Pedagógicos, 36(2), 333-342. https:// doi.org/10.4067/s0718-07052010000200019 
Galston, W. (1991). Liberal purposes. Good, virtues, and duties in the liberal state. Cambridge: Cambridge University Press. https://doi.org/10.1017/ CBO9781139172462

Garretón, M.A. (2010). La democracia incompleta en chile: la realidad tras los rankings internacionales. Revista de Ciencia Politica, 30(1), 115-148.

Glatthorn, A., Boschee, F. y Whitehead, B. (2006). Curriculum leadership. Development and implementation. California: Sage.

Giddens, A. (1993). Consecuencias de la Modernidad. Madrid: Alianza Editorial.

González-Valencia, G. y Santisteban-Fernández, A. (2016). La formación ciudadana en la educación obligatoria en Colombia: entre la tradición y la transformación. Educación y educadores, 19(1), 89-102. https://doi.org: 10.5294/edu.2016.19.1.5

Kramer, S. (2005). Infancia, cultura y currículo: desafíos para la escuela. Educación, $14(27), 33-50$.

Kymlicka, W., y Norman, W. (2002). El retorno del ciudadano. Una revisión de la producción reciente en teoría de la ciudadanía. Lima: Instituto de Estudios Peruanos.

Labarca, A. (1944). La educación como función social. En Bases para una política educacional (pp.9-18). Santiago de Chile: Biblioteca Fundamentos de la Construcción de Chile, Pontifica Universidad Católica.

Mardones, R. (2015). El paradigma de la educación ciudadana en Chile: una política pública inconclusa. En C. Cox y J.C. Castillo (Eds.), Aprendizajes de la ciudadanía. Contextos, experiencias y resultados (pp.145-173). Santiago de Chile: Ediciones Universidad Católica de Chile.

Martuccelli, D. y de Singly, F. (2012). Las sociologías del individuo. Santiago de Chile: LOM.

Mineduc (2016). Orientaciones para la elaboración del Plan de Formación Ciudadana. Ministerio de Educación. Recuperado de http://www.mineduc. cl/wp-content/uploads/sites/19/2016/04/DEG-OrientacionesPFCintervenible-AReader_FINAL.pdf

Monzón, Y. (2015). Una política educativa: la equidad. Revista Iberoamericana para la Investigación y el Desarrollo Educativo (RIDE), 5(10). https://doi. org/10.23913/ride.v5i10.110

Mucchielli, A. (2011). Diccionario de Métodos cualitativos en ciencias humanas y sociales. Madrid: Editorial Síntesis.

Muñoz, M.L., López, M., y Assaél, J. (2015). Concepciones docentes para responder a la diversidad: ¿Barreras o recursos para la inclusión educativa? Revista Psicoperspectivas, 14(3), 68-79. https://doi.org/10.5027/ psicoperspectivas-Vol14-Issue3-fulltext-646 
Norris, P. (2003). Young people and political activism: From the politics of loyalties to the politics of choice? En Report for the Council of European Symposium. 'Young people and democratic institutions: From disillusionment to participation'. Recuperado de https://sites.hks.harvard.edu/fs/pnorris/Acrobat/ COE\%20Young\%20People\%20and\%20Political\%20Activism.pdfg

Pérez, A. (2000). La cultura escolar en la sociedad neoliberal. Madrid: Morata.

Pinar, W. (2014). La teoría del currículum. Madrid: Narcea.

Retamozo, M. (2009). Lo político y la política: los sujetos políticos, conformación y disputa por el orden social. Revista Mexicana de Ciencias Politicas y Sociales, 51(206), 69-91.

Reyes-Jedlicki, L., Miranda, C., Santa Cruz, E., Cornejo, R., Núnez, M., Arévalo, A., Hidalgo, F. (2014). Subjetividades y saberes docentes en el sistema educativo chileno: un análisis de las concepciones de formadores de profesores. Estudios Pedagógicos, 40(1), 183-203. https://doi.org/10.4067/ S0718-07052014000200011

Rojas, N., y Silva, C. (2016). La migración en Chile. Breve reporte y caracterización. Informe Observatorio Iberoamericano sobre Movilidad Humana, Migraciones y Desarrollo. Recuperado de http://www.extranjeria.gob.cl/ media/2016/08/informe_julio_agosto_2016.pdf

Salazar, G., y Pinto, J. (2010). Historia contemporánea de Chile. Tomo I. Santiago: LOM.

Schulz, W., Ainley, J., Frailon, J., Kerr, D. y Losito, B. (2010). ICCS 2009 International Report: Civic knowledge, attitudes, and engagement among lower-secondary school students in 38 countries. International Association for the Evaluation of Educational Achievement. Amsterdam: Author.

Silva, M. (2008). Currículum y Educación Intercultural. En M. Silva (Comp.), Nuestras Universidades y la educación intercultural (pp. 97-103). Santiago de Chile: Universidad de Chile.

Sloam, J. (2016). Diversity and voice: The political participation of young people in the European Union. British Journal of Politics \& International Relations, 18(3), 521-537. https://doi.org/10.1177/1369148116647176

Tijoux, M.E., y Córdova, M.G. (2015). Racismo en Chile: colonialismo, nacionalismo, capitalismo. Revista Polis, 14(42), 7-13. https://doi.org/10.4067/ S0718-65682015000300001

Touraine, A. (2001). ¿Podemos vivir juntos? Iguales y diferentes. México: FCE.

United Nations (2016). International migration report 2015. Department of Economic and Social Affairs. Nueva York: United Nations. 